\title{
Randomized trial of amino acid mixture combined with physical activity promotion for abdominal fat reduction in overweight adults
}

This article was published in the following Dove Press journal: Diabetes, Metabolic Syndrome and Obesity:Targets and Therapy

\author{
Keisuke Ueda ${ }^{1,2}$ \\ Hiroyuki Sasai ${ }^{3}$ \\ Takehiko Tsujimoto ${ }^{4}$ \\ Chiaki Sanbongi' \\ Shuji Ikegami' \\ Hiroyuki Kobayashi ${ }^{5}$ \\ Nobuhiko Shioya ${ }^{6}$ \\ Satoru Suzuki ${ }^{7}$ \\ Yoshio Nakata $^{5}$ \\ 'Food Science and Technology \\ Research Laboratories, Meiji Co., \\ Ltd, Hachiouji, ${ }^{2}$ Graduate School of \\ Comprehensive Human Sciences, \\ University of Tsukuba, Tsukuba, \\ ${ }^{3}$ Department of Life Sciences, \\ Graduate School of Arts and \\ Sciences, The University of Tokyo, \\ Tokyo, ${ }^{4}$ Faculty of Human Sciences, \\ Shimane University, Matsue, ${ }^{5}$ Faculty \\ of Medicine, University of Tsukuba, \\ Tsukuba, ${ }^{6} \mathrm{KSO}$ Corporation, Tokyo, \\ ${ }^{7}$ Shinagawa Season Terrace Health \\ Care Clinic, Tokyo, Japan
}

Purpose: The purpose of this study was to test the efficacy of arginine, alanine, and phenylalanine mixture (A-mix) ingestion at $1,500 \mathrm{mg}$ /day in combination with the promotion of physical activity for abdominal fat reduction in overweight adults.

Methods: A placebo-controlled, double-blind, parallel-group, randomized trial for 12 weeks combined with a 4-week follow-up period was conducted at a single center in Minato-ku, Tokyo, Japan, between December 2016 and May 2017. Data were analyzed between June and August 2017. The study participants were 200 overweight adults within the age range of 20-64 years. The participants were randomly assigned to the A-mix group $(n=100)$ or a placebo group $(\mathrm{n}=100)$ and were administered $500 \mathrm{~mL}$ of test beverage containing 1,500 or $0 \mathrm{mg}$ of A-mix, respectively, for 12 weeks. All participants maintained a physically active lifestyle between week 0 and week 12 through monthly sessions of physical activity. The primary outcomes were the 12 -week changes in the abdominal total, subcutaneous, and visceral fat areas, as assessed by computed tomography.

Results: Of the 200 enrolled participants, 199 (99\%) accomplished the 12-week intervention and 4-week follow-up period. The per-protocol-based analysis for 194 participants demonstrated that the abdominal total fat area decreased significantly in the A-mix group compared with that in the placebo group (difference, $10.0 \mathrm{~cm}^{2} ; 95 \%$ confidence interval [CI]: $0.4-19.6 \mathrm{~cm}^{2} ; P=0.041$ ). Comparable outcomes were obtained for the abdominal subcutaneous fat area (difference, 7.4 $\mathrm{cm}^{2} ; 95 \% \mathrm{CI}: 0.1-14.7 \mathrm{~cm}^{2} ; P=0.047$ ). No study-related unfavorable events occurred.

Conclusion: A-mix supplementation in combination with physical activity promotion facilitated abdominal fat reduction in overweight adults.

Keywords: amino acid supplementation, physical activity promotion, abdominal fat, randomized controlled trial

\section{Introduction}

Obesity, which is a common medical issue worldwide, ${ }^{1}$ is a major risk factor for type 2 diabetes, insulin resistance, atherosclerosis, hypertension, stroke, and several types of cancer. ${ }^{2}$ The condition results from a chronic imbalance in energy metabolism. To avoid or alleviate the risks associated with obesity, weight reduction by dietary restriction and a general exercise program is viewed as the best, accessible, nonpharmacologic, and nonsurgical treatment system. ${ }^{3}$

The additive effects or synergistic impact of eating routines and exercise have recently been thoroughly studied. ${ }^{4,5}$ Our research group has considered the impact of amino acid supplementation, in combination with exercise, on fat oxidation for over two decades. ${ }^{6-15}$ Recently, we concentrated on the amino acids arginine, alanine, and
Correspondence: Yoshio Nakata Faculty of Medicine, University of Tsukuba, I-I-I Tennodai, Tsukuba, Ibaraki 305-8575, Japan

$\mathrm{Tel} / \mathrm{Fax}+8$ I 298533076

Email nakata@md.tsukuba.ac.jp 
phenylalanine, which are involved in glucagon synthesis and secretion when delivered acutely. We also established that a single session of aerobic exercise in conjunction with oral administration of a mixture of arginine, alanine, and phenylalanine (A-mix) increased blood ketone body levels compared with placebo in humans. ${ }^{13,14}$ These conclusions indicated a lipolytic effect during exercise that was likely a result of A-mix ingestion. Moreover, we recently conducted a pilot randomized controlled trial to evaluate the long-term efficacy of A-mix consumption in conjunction with physical activity promotion for overweight individuals, and this trial helped to estimate the effective dose of the mixture. The pilot trial suggested that a dose of 1,500 $\mathrm{mg}$ /day of A-mix facilitated abdominal fat reduction in overweight adults. ${ }^{16}$

Therefore, we conducted a randomized controlled trial to confirm the efficacy of the combination of $1,500 \mathrm{mg} /$ day A-mix ingestion with physical activity promotion in abdominal fat reduction in overweight adults between the age of 20 and 64 years.

\section{Methods}

\section{Trial design}

The study had a double-blind, placebo-controlled, parallelgroup, and randomized design. The trial was a single-center study organized at a site in Minato-ku, Tokyo, Japan, and was conducted between December 2016 and May 2017. The data were analyzed between June and August 2017. The target number of participants was set at 200 (i.e., 100 participants per group), as a sample size of 100 participants in each group enabled us to perceive a group difference effect size (Cohen's $d$ ) of 0.4 with a significance level of $5 \%$ and a power of $80 \%$. Given the assumption that some individuals would refuse to participate in the trial or fail to meet the inclusion criteria, a target number of 340 adults was set for the introductory session. The investigation protocol was approved by the Institutional Review Board of the University of Tsukuba Faculty of Medicine, the Meiji Institutional Review Board, and the Ethical Committee of Nihonbashi Cardiology Clinic. Written informed consent was obtained from each participant before randomization. The study protocol was enrolled in the UMIN Clinical Trials Registry (UMIN000025186) on December 10, 2016. This article completely conforms to the Consolidated Standards of Reporting Trials 2010 rules. ${ }^{17}$ A data monitoring group guaranteed the accuracy of information collected and the sources of information.

\section{Participants}

Participants were recruited from a volunteer database associated with a contract research organization. The included participants had to be overweight $\left(25 \mathrm{~kg} / \mathrm{m}^{2} \leq\right.$ body mass index $[\mathrm{BMI}]<30 \mathrm{~kg} / \mathrm{m}^{2}$ ) adults between 20 and 64 years of age. Individuals were excluded if they 1) continually used oral medication that might conceivably influence the body weight and lipid metabolism; 2) had food allergies or cardiovascular, severe hepatic, respiratory, metabolic, or endocrine disorders; 3 ) could not avoid the use of health food products or supplements that might conceivably influence the body weight and lipid metabolism; 4) had a current or previous history of drug or alcohol dependence; 5) could not abstain from alcohol consumption for 2 days before each measurement visit; 6) had metal installed in their abdominal site; 7) had a cardiac pacemaker or an implanted defibrillator; 8) were diagnosed with hyperphenylalaninemia or phenylketonuria; 9) had a history of familial hyperlipidemia; 10) were currently pregnant, nursing, or intended to become pregnant during the study period; 11) had extremely irregular dietary propensities or lifecycle, for example, midnight working; 12) participated or were eager to participate in clinical trials of other foods, medications, or beauty products; 13) had a fear of confined places; and 14) were judged as inappropriate for the study by the principal investigator owing to abnormal blood or urine parameters, or other reasons.

\section{Randomization and blinding}

After arrangement by sex and intervention waves, the participants who met the inclusion criteria were randomized to the A-mix or placebo group with a 1:1 allocation ratio. An investigator who had no contact with the participants and staff individuals created a random number sequence by using an approved computer program. The random sequence was kept by an assigned individual who was accountable for shipping the test beverages to the participants but was not involved in the plan, enrollment, evaluation, intervention, or analysis. The participants, investigators, and all staff members involved in the trial were blinded to the group allocation. The randomization code was opened after the study information was checked, collated, and finalized.

\section{Interventions}

\section{Test beverage}

The test preparation for the A-mix and placebo groups consisted of a $500 \mathrm{~mL}$ polyethylene terephthalate bottle beverage that contained 1,500 and $0 \mathrm{mg}$ of A-mix, respectively. The 
test beverages had an identical appearance and comparable tangible attributes. The ingredients of the A-mix were as follows: $42 \% \mathrm{~mol} / \mathrm{mol}$ of phenylalanine, $38 \% \mathrm{~mol} / \mathrm{mol}$ of alanine, and $20 \% \mathrm{~mol} / \mathrm{mol}$ of arginine. Each bottle of the test beverage contained 20 and $13 \mathrm{kcal}$ for the A-mix and the placebo groups, respectively. Neither of the test beverages contained caffeine. The assigned individual transported the test beverages to the participants based on group allocation. The participants were instructed to keep the test beverages away from high humidity, high temperature, and direct sunlight and to consume one bottle per day before and during physical activity or exercise under free-living conditions.

\section{Physical activity promotion}

Regardless of group allocation, the participants followed a monthly physical activity promotion session throughout the 12-week intervention period (four sessions in total). The intervention program consisted of a group-based exercise class with personalized advice. Participants were urged to add 1,000 steps/day or more from their benchmark step targets suggested by the current Japanese physical activity recommendations. ${ }^{18,19}$ The group-based exercise class started with a 30 min lecture and a 60 min group-based workout session that included walking, muscle strengthening, and stretching. Trained professionals conducted the personalized advice sessions in conjunction with the analysis of participants' recent step counts, body weight, test beverage ingestion, physical condition, medication use, dietary behaviors, daily step counts measured by pedometer (FB-736; Tanita Corporation, Tokyo, Japan), and exercise, all of which were recorded in diaries. To avoid the confounding effects of lifestyle changes, participants were instructed to consume well-balanced meals three times per day in accordance with the Japanese Food Guide Spinning Top ${ }^{20}$ and to abstain from excessive strenuous exercise, gorging, serious activity restrictions, ingestion of healthful supplements, functional foods, and over-the-counter medications that could possibly influence the results.

\section{Measurements}

All study outcomes, except for abdominal fat area, were assessed at baseline and at each visit after 4 weeks by experienced staff members who were blinded to the group allocation. The abdominal fat areas were scanned by using computed tomography (CT) at the baseline and 12-week visits. The primary outcome measures were the 12-week changes in the abdominal fat areas. The secondary outcomes comprised changes in body weight, percent fat mass, waist and hip circumferences, and metabolic risk factors such as lipid profiles, blood glucose parameters, and blood pressure. To monitor adherence to the intervention, dietary intake was measured every 4 weeks and physical activity was measured at baseline and week 12 .

\section{Anthropometrics and body composition}

Body weight (to the nearest $0.1 \mathrm{~kg}$ ) and percent fat mass (to the nearest $0.1 \%$ ) were measured by using a multi-frequency bioelectrical impedance device (InBody 430; Biospace, Seoul, Korea). ${ }^{21}$ Height was measured to the nearest $0.1 \mathrm{~cm}$ by utilizing a compact stadiometer (DSN-90; Muratec-KDS Corp., Kyoto, Japan) at baseline. BMI was estimated from participants' weight in kilograms divided by their height in meters squared. Waist and hip circumference were measured twice to the nearest $0.1 \mathrm{~cm}$ at the umbilicus and maximum circumference of buttocks, respectively in the standing position by using a flexible plastic tape. The average value of the two measurements was used for analysis. Waist to hip ratio was calculated from participants' waist circumference divided by their hip circumference.

\section{Abdominal fat areas}

The abdominal fat areas were analyzed at the L4 level by using CT (Supria; Hitachi Ltd, Tokyo, Japan) and computed by a commercial program (Fat Scan Ver. 5.0.; e-JAPAN IT Co., Ltd, Ibaraki, Japan). ${ }^{22}$

\section{Blood pressure readings}

Systolic and diastolic blood pressure readings were measured with a mechanized sphygmomanometer (Digital Automatic Blood Pressure Monitor HEM-907; Omron Healthcare, Kyoto, Japan). The measurements were taken on the arm of seated participants who had rested for no less than $5 \mathrm{~min}$ with the arm supported at the heart level. An average value of both readings was used for data analysis.

\section{Blood biochemistry}

A blood sample was drawn from each participant after an overnight ( $\geq 12 \mathrm{~h}$ ) fast. Venous blood was assayed by an independent laboratory (LSI Medience Corporation, Tokyo, Japan). The levels of serum total cholesterol (IatoroLQ $\mathrm{T}-\mathrm{CHO}(\mathrm{A}) \mathrm{II}$; LSI Medience Corporation) and triglycerides (IatoroLQ TG II; LSI Medience Corporation) were determined enzymatically. Serum high- and low-density lipoprotein cholesterol levels were measured by the selective inhibition method (MetaboLead HDL-C and MetaboLead LDL-C, respectively; Kyowa Medex Co., Ltd, Tokyo, Japan). Blood glucose was assayed by an enzymatic method 
(IatoroLQ GLU; Unitika, Aichi, Japan) and insulin by a chemiluminescent immunoassay (Architect Insulin; Abbott Japan, Tokyo, Japan). Glycated hemoglobin A $\left(\mathrm{HbA}_{1 \mathrm{c}}\right)$ was determined enzymatically (CinQ HbAlc; ARKRAY Inc., Kyoto, Japan). Homeostasis model assessment of insulin resistance (HOMA-R) was calculated using the following formula: Blood glucose $(\mathrm{mg} / \mathrm{dL}) \times$ insulin $(\mu \mathrm{U} / \mathrm{mL}) / 405$.

\section{Dietary intake}

The macronutrient intake in grams and the total energy intake in kilocalories were evaluated from records of food intake over 3 days. Participants were instructed to record their total food consumption for 3 days, including two weekdays and one weekend day. Food quantities were measured by using standard measuring glasses, spoons, and digital scales.

\section{Physical activity}

Physical activity was measured by using a triaxial accelerometer (Active style Pro HJA-750C; Omron Healthcare) worn at the waist for 7 days. The accelerometer counted steps and computed the intensity of physical activity (expressed as metabolic equivalents) from a published algorithm. ${ }^{23,24}$ The devices were not worn during sleeping, water-based activities (e.g., washing or swimming), or participating in certain exercises for safety reasons (e.g., contact sports). Records obtained were characterized as substantial when a device was worn for no less than $10 \mathrm{~h} /$ day. ${ }^{25}$ When substantial records were gathered for over 1 day, step counts and time spent in moderate to vigorous physical activity ( $\geq 3$ metabolic equivalents) were calculated for each participant.

\section{Safety assessment}

Hematology tests, blood biochemical tests, and urinalyses were performed at every visit after 4 weeks. At the same visit, a medical staff checked the health status of the participants by means of a medical interview and consultation with their diaries.

\section{Adverse events}

Data on adverse events were subjectively obtained through the participants' diaries and were independently analyzed by using pulse rate measurements and blood hematologic and biochemical tests. In this trial, an adverse event was characterized as any negative and unintended sign, manifestation,

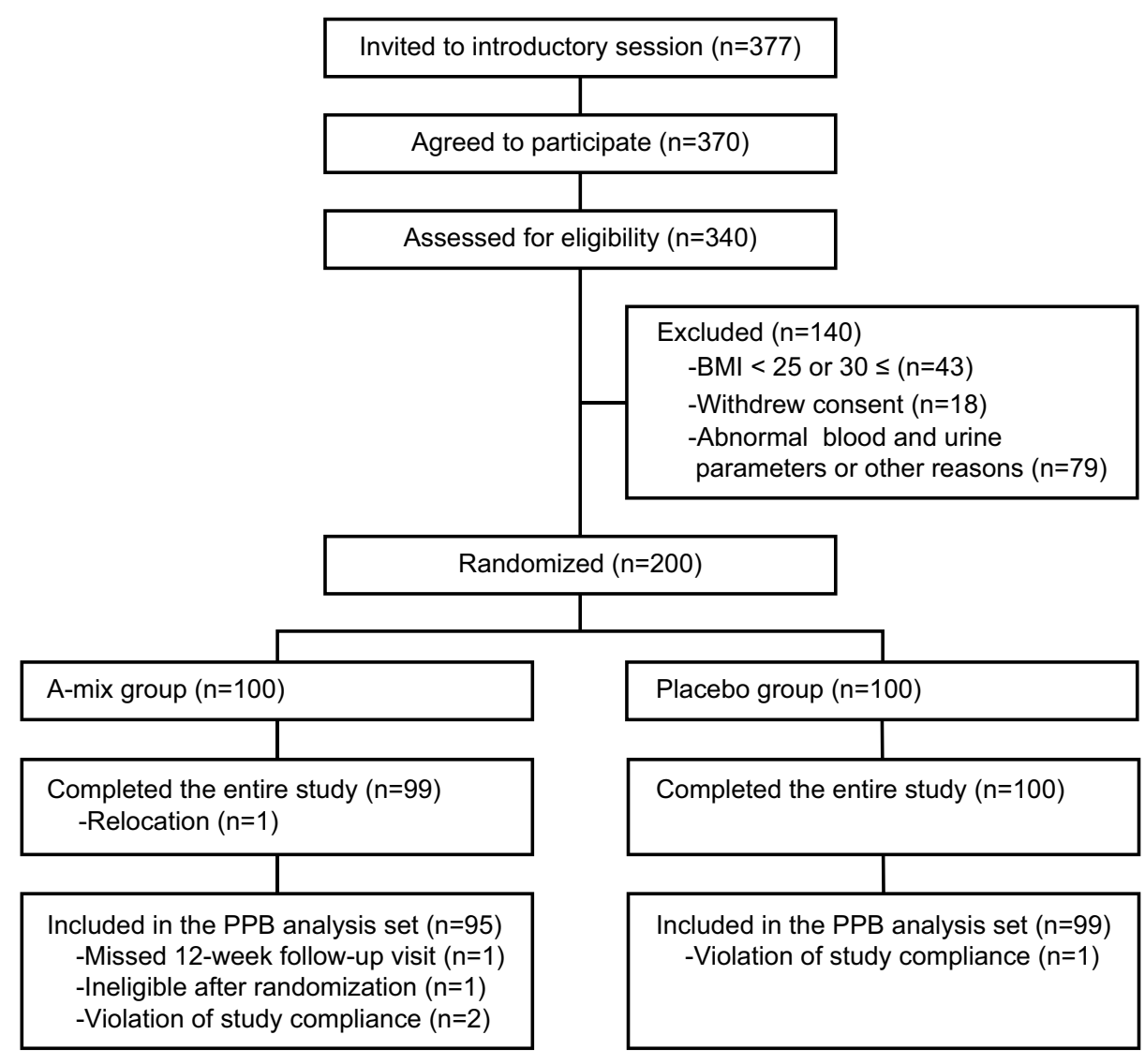

Figure I Flowchart of trial participants.

Abbreviations: BMI, body mass index; PPB, per-protocol-based. 
or malady. The study physician then judged whether a given unfavorable event was serious or nonserious, and if it was study related.

\section{Statistical analysis}

All statistical analyses were performed in accordance with the analysis plan in the study protocol using IBM SPSS Statistics Ver. 24 (IBM Japan, Ltd, Tokyo, Japan). Values of $P<0.05$ were considered statistically significant. The baseline participant characteristics were presented as the mean and standard deviation for continuous variables or as frequencies and percentages for categorical variables. Our primary analysis followed a per-protocol-based (PPB) principle, in which we excluded participants who met no less than one of the accompanying predetermined criteria as follows: 1) missed the 12-week visit; 2 ) low compliance rate $(<80 \%)$ for consumption of the test beverages; 3 ) absent or inadequate diary logs; 4) announced ineligible after randomization; and 5) serious infringement of study consistence, as judged by the principal investigator.

An unpaired Student's $t$-test was used to compare the 12-week changes in the primary, secondary, and compliance outcomes. A stratified analysis was conducted: those with step counts increased by 1,000 steps or more and those with step counts increased by fewer than 1,000 steps during the 12-week intervention period. In the safety assessment, the adverse events and deviations identified by fasting blood tests were compared between the two groups by using chisquare tests for categorical variables and unpaired $t$-tests for continuous variables. In addition, paired $t$-tests were used to compare the data before and after the intervention within each group.

\section{Results}

The complete flow chart of the study participants is displayed in Figure 1. A total of 377 candidates attended the introductory session; written informed consent was obtained from 370 participants and the baseline examination was completed by 340 participants. Finally, 200 individuals met the inclusion criteria and were assigned randomly to the A-mix group $(n=100)$ or to the placebo group $(n=100)$.

The characteristics of the participants measured at baseline are presented in Table 1. There were no significant differences in any study or compliance outcomes between the two groups. Among the 200 participants, 199 (99\%) completed the 12-week intervention and 4-week follow-up period; of the 199 who completed the trial, the median rate of test beverage intake was $100 \%$ with no between-group
Table I Baseline characteristics of the participants $(n=200)$

\begin{tabular}{|c|c|c|}
\hline Characteristic & $\begin{array}{l}\text { A-mix group } \\
(n=100)\end{array}$ & $\begin{array}{l}\text { Placebo group } \\
(n=100)\end{array}$ \\
\hline Female, n (\%) & $50(50)$ & $50(50)$ \\
\hline Age, years & $43.5(9.9)$ & $43.5(9.7)$ \\
\hline Height, cm & $164.9(9.2)$ & I64.3 (8.2) \\
\hline Weight, kg & $73.5(9.1)$ & $73.4(7.8)$ \\
\hline Body mass index, $\mathrm{kg} / \mathrm{m}^{2}$ & $27.0(1.2)$ & $27.1(1.3)$ \\
\hline Percent fat mass, $\%$ & $32.1(6.9)$ & $32.4(6.9)$ \\
\hline Waist circumference, $\mathrm{cm}$ & $93.8(5.8)$ & $93.6(4.9)$ \\
\hline Hip circumference, cm & $101.2(4.2)$ & $100.5(3.8)$ \\
\hline Waist to hip ratio & $0.93(0.05)$ & $0.93(0.05)$ \\
\hline \multicolumn{3}{|l|}{ Abdominal fat area, $\mathrm{cm}^{2}$} \\
\hline Total & $339.2(73.4)$ & $327.6(71.1)$ \\
\hline Subcutaneous & $244.0(66.2)$ & $229.7(70.4)$ \\
\hline Visceral & $95.2(35.9)$ & $98.0(38.9)$ \\
\hline \multicolumn{3}{|l|}{ Blood pressure, mm Hg } \\
\hline Systolic & $129.8(13.6)$ & $129.4(12.5)$ \\
\hline Diastolic & $77.6(10.4)$ & $77.5(10.6)$ \\
\hline \multicolumn{3}{|l|}{ Blood biochemical parameters } \\
\hline Triglycerides, mg/dL & I03.I (53.8) & II $3.4(55.5)$ \\
\hline Total cholesterol, mg/dL & $208.3(33.5)$ & $215.0(30.2)$ \\
\hline $\mathrm{HDL}-\mathrm{C}, \mathrm{mg} / \mathrm{dL}$ & $58.3(13.8)$ & $56.4(11.3)$ \\
\hline LDL-C, mg/dL & 126.I (30.6) & $133.6(27.1)$ \\
\hline Fasting blood glucose, mg/dL & $85.1(6.2)$ & $85.3(7.4)$ \\
\hline Glycated hemoglobin $A_{1 c}, \%$ & $5.5(0.3)$ & $5.5(0.3)$ \\
\hline Fasting insulin, $\mu \mathrm{U} / \mathrm{mL}$ & $5.6(2.7)$ & $5.8(2.4)$ \\
\hline HOMA-R & $1.18(0.61)$ & $1.22(0.53)$ \\
\hline \multicolumn{3}{|l|}{ Dietary intake } \\
\hline Total energy, kcal/day & $\mathrm{I}, 934(47 \mathrm{I})$ & $1,850(393)$ \\
\hline Protein, g/day & $72.4(21.0)$ & $68.1(16.0)$ \\
\hline Fat, g/day & $67.1(22.0)$ & $63.0(17.0)$ \\
\hline Carbohydrate, g/day & $243.8(62.0)$ & $238.5(57.1)$ \\
\hline \multicolumn{3}{|l|}{ Physical activity } \\
\hline Step counts, steps/day* & $7,565(3,308)$ & $8,454(3,598)$ \\
\hline MVPA, $\min / d a y^{*}$ & $65.5(32.7)$ & $70.2(37.2)$ \\
\hline
\end{tabular}

Notes: Data shown as mean (standard deviation) unless specified. *Data were available for 198 participants (100 and 98 in the A-mix and the placebo groups, respectively).

Abbreviations: HDL-C, high-density lipoprotein cholesterol; LDL-C, low-density lipoprotein cholesterol; HOMA-R, homeostasis model assessment of insulin resistance; MVPA, moderate-to-vigorous physical activity.

difference ( $P=0.42$; Mann-Whitney $U$ test $)$. After the exclusion of five additional participants (1 missed the 12-week visit, 1 was ineligible after randomization, and 3 seriously violated the study compliance requirements), 194 participants were included in the PPB analysis.

The results of the PPB analyses of the primary and secondary outcomes are presented in Table 2 and Figure 2 , and those of the compliance outcomes are summarized in Table 3. The abdominal total fat area decreased significantly in the A-mix group compared with that in the placebo group (difference, $10.0 \mathrm{~cm}^{2} ; 95 \%$ confidence interval [CI]: $\left.0.4-19.6 \mathrm{~cm}^{2} ; P=0.041\right)$. Similar results were obtained for the abdominal subcutaneous fat area measurements (difference, $7.4 \mathrm{~cm}^{2} ; 95 \%$ CI: $\left.0.1-14.7 \mathrm{~cm}^{2} ; P=0.047\right)$. No noteworthy 
Table 2 Per-protocol-based analyses for primary and secondary outcomes $(n=194)$

\begin{tabular}{|c|c|c|c|c|c|c|}
\hline \multirow[t]{2}{*}{ Outcome } & \multicolumn{6}{|c|}{ A-mix group $(n=95)$} \\
\hline & Week 0 & Week 4 & Week 8 & Week I2 & Week 16 & I2-week change \\
\hline \multicolumn{7}{|l|}{ Primary outcomes } \\
\hline Abdominal total fat area, $\mathrm{cm}^{2}$ & $338.8(73.6)$ & NA & NA & $320.5(76.1)$ & NA & $-18.3(-25.8,-10.8)$ \\
\hline Abdominal subcutaneous fat area, $\mathrm{cm}^{2}$ & $245.2(66.1)$ & NA & NA & $236.3(68.1)$ & NA & $-8.9(-14.5,-3.3)$ \\
\hline Abdominal visceral fat area, $\mathrm{cm}^{2}$ & $93.6(35.6)$ & NA & NA & $84.1(34.0)$ & NA & $-9.5(-12.9,-6.0)$ \\
\hline \multicolumn{7}{|l|}{ Secondary outcomes } \\
\hline Weight, kg & $73.6(8.9)$ & $73.2(8.7)$ & $73.0(8.8)$ & $72.4(8.7)$ & $72.6(8.8)$ & $-1.2(-1.7,-0.8)$ \\
\hline Body mass index, $\mathrm{kg} / \mathrm{m}^{2}$ & $27.1(1.2)$ & $27.0(1.2)$ & $26.9(1.2)$ & $26.7(1.3)$ & $26.7(1.3)$ & $-0.4(-0.6,-0.3)$ \\
\hline Percent fat mass, $\%$ & $31.9(7.0)$ & $32.1(7.4)$ & $32.1(7.4)$ & $30.7(7.5)$ & $31.4(7.7)$ & $-1.2(-1.6,-0.8)$ \\
\hline Waist circumference, $\mathrm{cm}$ & $94.0(5.4)$ & $93.8(5.4)$ & $93.0(5.2)$ & $92.6(5.4)$ & $92.3(5.7)$ & $-1.4(-1.9,-0.9)$ \\
\hline Hip circumference, cm & $100.8(4.3)$ & $100.7(4.0)$ & $100.4(4.0)$ & $100.0(4.1)$ & $100.0(4.2)$ & $-0.8(-1.2,-0.4)$ \\
\hline Waist to hip ratio & $0.93(0.04)$ & $0.93(0.04)$ & $0.93(0.04)$ & $0.93(0.04)$ & $0.92(0.04)$ & $-0.01(-0.01,0.00)$ \\
\hline Systolic blood pressure, $\mathrm{mm} \mathrm{Hg}$ & $124.0(15.1)$ & $126.1(15.7)$ & $122.0(14.3)$ & $115.2(14.8)$ & $121.9(16.6)$ & $-8.8(-11.0,-6.7)$ \\
\hline Diastolic blood pressure, $\mathrm{mm} \mathrm{Hg}$ & $79.5(11.5)$ & 76.9 (11.2) & $73.7(10.9)$ & $70.5(9.5)$ & 73.7 ( 11.2$)$ & $-9.0(-10.7,-7.2)$ \\
\hline Triglycerides, mg/dL & $103.9(54.9)$ & $103.2(61.3)$ & $98.0(53.5)$ & $95.8(54.5)$ & I03.I (5I.8) & $-8.1(-18.2,2.1)$ \\
\hline Total cholesterol, mg/dL & $209.9(33.2)$ & $208.6(33.9)$ & $209.4(33.8)$ & $200.2(33.7)$ & $208.3(35.9)$ & $-9.7(-14.3,-5.0)$ \\
\hline $\mathrm{HDL}-\mathrm{C}, \mathrm{mg} / \mathrm{dL}$ & $58.5(13.9)$ & $57.0(13.3)$ & $58.0(13.9)$ & $56.0(12.8)$ & $56.7(13.1)$ & $-2.5(-3.7,-1.3)$ \\
\hline LDL-C, mg/dL & $127.4(30.8)$ & $127.3(32.4)$ & $126.0(30.8)$ & $119.9(30.7)$ & 126.I (32.2) & $-7.5(-11.4,-3.6)$ \\
\hline Fasting blood glucose, $\mathrm{mg} / \mathrm{dL}$ & $85.1(6.2)$ & $85.2(8.0)$ & $85.0(7.0)$ & $84.5(7.7)$ & $83.0(6.6)$ & $-0.6(-1.7,0.5)$ \\
\hline Glycated hemoglobin $A_{1 c}, \%$ & $5.47(0.29)$ & $5.5 \mathrm{I}(0.27)$ & $5.45(0.27)$ & $5.44(0.26)$ & $5.47(0.28)$ & $-0.03(-0.07,0.01)$ \\
\hline Fasting insulin, $\mu \mathrm{U} / \mathrm{mL}$ & $5.4(2.3)$ & $5.4(3.0)$ & $6.7(8.1)$ & $5.4(2.0)$ & $6.2(4.1)$ & $0.0(-0.5,0.4)$ \\
\hline HOMA-R & $\mathrm{I} .14(0.50)$ & $1.15(0.68)$ & $\mathrm{I} .43(1.85)$ & $1.13(0.45)$ & $1.28(0.91)$ & $-0.01(-0.12,0.09)$ \\
\hline
\end{tabular}

Notes: Data at weeks $0,4,8,12$, and 16 are presented as mean (standard deviation). Changes from baseline to week 12 are presented as mean (95\% confidence interval). $P$-value is a result of unpaired $t$-test for 12 -week change between groups.

Abbreviations: HDL-C, high-density lipoprotein cholesterol; LDL-C, low-density lipoprotein cholesterol; HOMA-R, homeostasis model assessment of insulin resistance; NA, not assessed.

difference between groups was found for the other primary and secondary outcomes. The daily total energy intake was decreased by $131 \mathrm{kcal}$ in the A-mix group and by $77 \mathrm{kcal}$ in the placebo group. The daily step count was increased by 1,779 in the A-mix group and by 1,629 in the placebo group. No significant between-group differences were found in the consistency of the outcome measurements.

In the stratified analysis (Table 4) among the participants whose step counts increased by more than 1,000 steps during the 12-week intervention period, the A-mix group showed a significantly larger reduction in the abdominal total and subcutaneous fat areas compared with the placebo group ( $P=0.019$ and 0.011 , respectively). However, no significant between-group differences were observed in the participants whose step counts increased by fewer than 1,000 steps during the intervention.

In the safety assessment, no serious, unfavorable events occurred in the trial. A total of 133 nonserious unfavorable events were accounted for through participants' diaries and medical interviews (Table S1). These occasions included fever, migraine, toothache, arthralgia, and common symptoms, for example, cold or sensitivity to dust. Although there were significant differences between the groups (e.g., allergy to pollen and allergic rhinitis), none of these were judged by the study physician to be related to the consumption of the study beverage. Moreover, a total of 456 deviations from the reference ranges were identified through blood analysis (Table S2). Although there were significant differences between groups, such as increased number of red blood cells, elevated levels of hemoglobin, decreased levels of aspartate amino transferase, decreased levels of alanine aminotransferase, elevated levels of total bilirubin, elevated levels of blood urea nitrogen, elevated levels of calcium, and elevated levels of serum iron, there were no clinically significant variations from the norm or discoveries, as judged by the study physician, due to the consumption of the test beverage on the basis of the physical assessments, blood biochemical analyses, and hematologic parameters. The results of the blood analyses and urinalyses are summarized in Tables S3-S6.

\section{Discussion}

The aim of this study was to test the efficacy of A-mix in conjunction with the promotion of physical activity for the reduction of abdominal fat in overweight adults. We found significantly larger reduction in abdominal total and subcutaneous fat areas in the A-mix group compared with the placebo group. From a safety perspective, no serious, 


\begin{tabular}{|c|c|c|c|c|c|c|}
\hline \multicolumn{6}{|c|}{ Placebo group $(n=99)$} & \multirow[t]{2}{*}{$P$-value } \\
\hline Week 0 & Week 4 & Week 8 & Week I2 & Week 16 & I 2-week change & \\
\hline $326.2(70.0)$ & NA & NA & $317.8(71.3)$ & NA & $-8.3(-14.5,-2.2)$ & 0.041 \\
\hline $228.3(69.4)$ & NA & NA & $226.8(69.1)$ & NA & $-1.5(-6.2,3.3)$ & 0.047 \\
\hline $97.9(39.0)$ & NA & NA & $91.0(38.8)$ & NA & $-6.8(-9.6,-4.1)$ & 0.24 \\
\hline $73.5(7.8)$ & $73.1(8.0)$ & 72.9 (7.9) & $72.3(7.8)$ & $72.4(7.8)$ & $-1.2(-1.7,-0.8)$ & 0.96 \\
\hline $27.2(1.3)$ & $27.0(1.4)$ & $26.9(1.5)$ & $26.7(1.5)$ & $26.8(1.6)$ & $-0.5(-0.6,-0.3)$ & 0.94 \\
\hline $31.8(6.8)$ & $32.2(7.2)$ & $32.0(7.0)$ & $30.6(7.1)$ & $31.3(7.4)$ & $-1.2(-1.6,-0.9)$ & 0.93 \\
\hline $94.0(5.4)$ & $93.9(5.4)$ & $93.4(5.4)$ & $92.8(5.5)$ & $92.5(5.5)$ & $-1.2(-1.8,-0.7)$ & 0.65 \\
\hline $100.2(3.6)$ & I00.I (3.6) & $99.8(3.7)$ & $99.6(3.7)$ & $99.4(3.7)$ & $-0.6(-0.9,-0.3)$ & 0.37 \\
\hline $0.94(0.05)$ & $0.94(0.05)$ & $0.94(0.05)$ & $0.93(0.05)$ & $0.93(0.05)$ & $-0.01(-0.01,0.00)$ & 0.93 \\
\hline $124.8(16.0)$ & $126.3(15.1)$ & I24.6 (I4.2) & $116.9(13.1)$ & I24.7 (13.6) & $-7.9(-10.2,-5.6)$ & 0.55 \\
\hline $81.3(12.5)$ & $77.8(11.3)$ & 75.5 (II.2) & $73.7(10.0)$ & $75.6(10.3)$ & $-7.5(-9.3,-5.8)$ & 0.26 \\
\hline II 3.1 (55.7) & II $9.0(58.1)$ & II $8.5(82.6)$ & II $0.9(88.0)$ & $110.4(51.7)$ & $-2.2(-20.0,15.5)$ & 0.57 \\
\hline $214.5(30.0)$ & $211.6(31.3)$ & $218.1(32.9)$ & $207.9(31.7)$ & $212.4(32.2)$ & $-6.6(-9.8,-3.4)$ & 0.28 \\
\hline $56.2(11.1)$ & $54.7(1 \mathrm{I} .0)$ & $56.0(12.1)$ & $54.9(11.2)$ & $55.3(11.7)$ & $-1.2(-2.6,0.1)$ & 0.17 \\
\hline I33.4 (27.2) & $129.6(27.1)$ & I32.6 (28.3) & I26.I (25.9) & |3I.I (28.4) & $-7.2(-10.0,-4.5)$ & 0.92 \\
\hline $85.2(7.4)$ & $86.3(7.0)$ & $86.3(9.3)$ & $84.1(7.7)$ & $83.6(6.8)$ & $-1.1(-2.4,0.1)$ & 0.53 \\
\hline $5.46(0.25)$ & $5.49(0.29)$ & $5.47(0.28)$ & $5.43(0.28)$ & $5.42(0.29)$ & $-0.03(-0.06,0.00)$ & 0.92 \\
\hline $5.8(2.4)$ & $5.9(3.7)$ & $7.1(4.2)$ & $5.8(2.8)$ & $6.5(2.6)$ & $0.1(-0.5,0.6)$ & 0.81 \\
\hline $1.22(0.53)$ & $1.27(0.86)$ & $1.53(0.94)$ & $1.23(0.65)$ & $\mathrm{I} .34(0.53)$ & $0.01(-0.12,0.15)$ & 0.76 \\
\hline
\end{tabular}

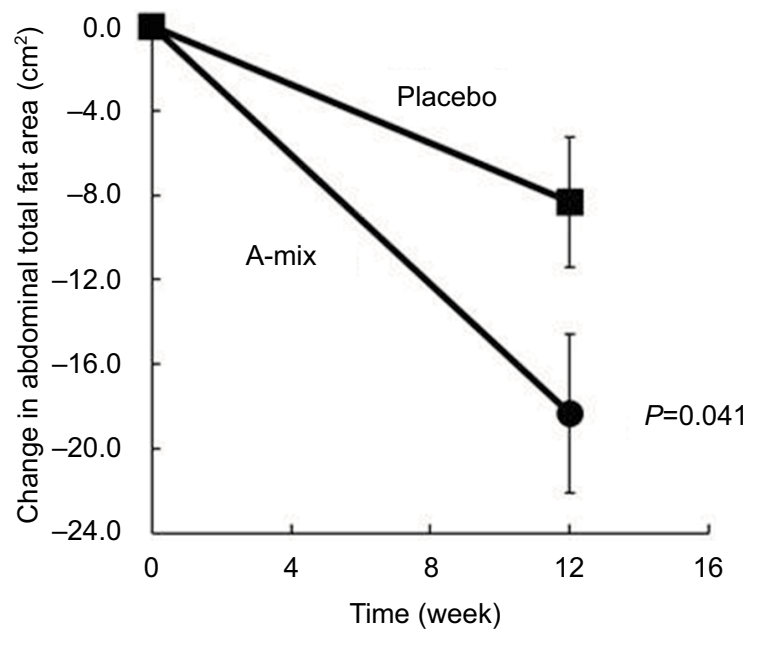

Figure 2 Pattern of change in abdominal total fat area during 12-week intervention by treatment assignment $(n=194)$.

Notes: Each data point represents the mean value for per-protocol-based analysis. Error bars indicate standard errors.

study-related, adverse events were identified throughout the examination. Although common symptoms, such as cold or allergy to pollen, and some abnormal lab tests of blood biochemistry were identified, these unfavorable events were independent of group assignment or were judged by the study physician to be not related to the consumption of the test beverage.
The larger reduction in abdominal fat in the A-mix group could be explained by the following physiological mechanisms described previously by our group. ${ }^{16}$ In brief, a previous study ${ }^{13}$ showed that pre-exercise ingestion of the A-mix was associated with increased blood concentrations of adrenaline and glucagon during and after exercise. Gannon and Nuttall reported that specific amino acids such as arginine, glycine, phenylalanine, and alanine can stimulate glucagon secretion. ${ }^{26}$ Therefore, the stimulation of glucagon secretion by the A-mix is characteristic of these amino acids. In this way, the reduction in blood glucose by exercise and a transient increase in blood amino acid levels by ingestion may stimulate pancreatic alpha cells and promote glucagon secretion. Additionally, past studies have noticed that some of the wide-ranging effects of adrenaline and glucagon appear to be facilitated by a common effector, cyclic adenosine $3^{\prime}, 5^{\prime}$-monophosphate. ${ }^{27}$ Therefore, cyclic adenosine $3^{\prime}, 5^{\prime}$-monophosphate-dependent lipolysis and fat oxidation appears to increase after a combination of the acute administration of A-mix and exercise, and the accumulated effects reduced abdominal fat in this study. However, the pathway described above is one of the mechanisms for the enhanced lipolysis and fat oxidation associated with A-mix supplementation combined with exercise. Moreover, 


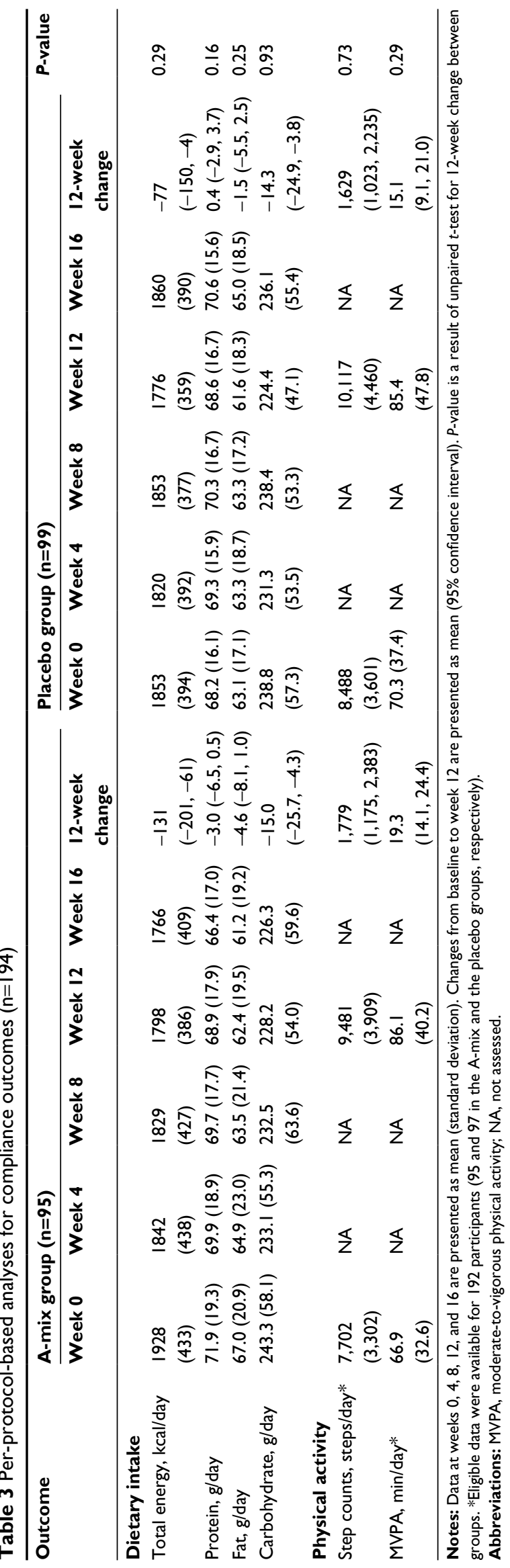

previous studies have suggested that glucagon secretion is regulated by gut hormones including glicentin, glucagon-like peptide-1, glucose-dependent insulinotropic polypeptide, and glucagon-like peptide- 2 and other peptides secreted by the gastrointestinal tract, such as gastrin-releasing peptide, cholecystokinin, and secretin. ${ }^{28}$ Therefore, future studies are necessary to investigate the mechanism for the reduction in abdominal fat area observed with A-mix supplementation combined with physical activity promotion.

Although many trials have reported that the long-term supplementation of amino acids, combined with exercise, increased fat mobilization and/or weight loss, ${ }^{29-36}$ this study focused on the promotion of physical activity, rather than a specific exercise training program. For example, Lucotti et al focused on the effects of $8.3 \mathrm{~g}$ arginine supplementation combined with two 45 min sessions per day of whole body exercise five times per week for 5 weeks. ${ }^{33}$ Ispoglou et al focused on the effects of $4.0 \mathrm{~g}$ leucine supplementation combined with a resistance training program using eight standard exercise machines twice per week for 12 weeks. ${ }^{35}$ These prescribed exercise programs were more intensive than that used in this study. Low-to-moderate intensity exercise may be better than vigorous exercise for the promotion of fat mass reduction, because the substrate oxidation achieved during high-intensity exercise primarily involves carbohydrate oxidation. ${ }^{37}$ In addition, the encouragement of moderate-intensity exercise is more feasible and acceptable than that of high-intensity physical activity in patients with obesity. Therefore, the benefits of A-mix intake combined with mild physical activity can be applied to wider range of populations (eg older adults) than those with previous studies. Further studies are needed to address this hypothesis.

The safety of the A-mix has been demonstrated by a single-dose toxicity test, a 91-day repeated-dose toxicity test, and Ames test. ${ }^{38-40}$ In this trial, some adverse events were found. Generally, these symptoms frequently occur after beverage ingestion and exercise, particularly among previously inactive individuals. Hence, the A-mix dose used in this study is unlikely to cause a serious safety concern for long-term ingestion. Several reports confirm the amino acid imbalance between aromatic amino acids (tyrosine, phenylalanine, and tryptophan) and branched-chain amino acids (BCAAs: valine, leucine, isoleucine), which leads to a decreased Fischer's ratio (BCAAs/aromatic amino acids) in both experimental models and clinical liver failure. ${ }^{41}$ However, indicators of liver function, such as aspartate aminotransferase, alanine aminotransferase, and $\gamma$-glutamyl transferase, were similar between the groups throughout the 
Table 4 Stratified analyses: participants whose step counts increased by I,000 steps or more $(n=109)$ and increased by fewer than $\mathrm{I}, 000$ steps $(\mathrm{n}=83)$ during the 12 -week intervention period

\begin{tabular}{|c|c|c|c|c|c|c|c|}
\hline & Week 0 & Week I 2 & I 2-week change & Week 0 & Week 12 & I 2-week change & $P$-value \\
\hline \multicolumn{8}{|c|}{ Participants whose step counts increased by more than I,000 steps in 12 weeks } \\
\hline & \multicolumn{3}{|c|}{ A-mix group $(n=6 I)$} & \multicolumn{4}{|c|}{ Placebo group $(n=48)$} \\
\hline Abdominal total fat area, $\mathrm{cm}^{2}$ & $330.1(79.0)$ & $304.6(80.2)$ & $-25.5(-35.6,-15.4)$ & $319.5(69.4)$ & $311.1(69.8)$ & $-8.4(-18.3,1.4)$ & 0.019 \\
\hline $\begin{array}{l}\text { Abdominal subcutaneous fat } \\
\text { area, } \mathrm{cm}^{2}\end{array}$ & $231.3(68.3)$ & $217.7(68.8)$ & $-13.6(-20.7,-6.5)$ & $219.2(68.7)$ & $218.6(67.9)$ & $-0.6(-7.5,6.3)$ & 0.011 \\
\hline Abdominal visceral fat area, $\mathrm{cm}^{2}$ & $98.8(37.1)$ & $86.9(34.2)$ & $-11.9(-16.7,-7.1)$ & $100.3(38.0)$ & $92.5(39.0)$ & $-7.8(-12.5,-3.1)$ & 0.23 \\
\hline Weight, kg & $74.6(9.0)$ & $72.9(8.7)$ & $-1.7(-2.3,-1.1)$ & $74.0(7.9)$ & $72.5(7.9)$ & $-1.4(-2.0,-0.9)$ & 0.51 \\
\hline Body mass index, $\mathrm{kg} / \mathrm{m}^{2}$ & $27.0(1.2)$ & $26.4(1.3)$ & $-0.6(-0.8,-0.4)$ & $27.1(1.3)$ & $26.5(1.5)$ & $-0.5(-0.7,-0.3)$ & 0.52 \\
\hline Percent fat mass, $\%$ & $30.5(6.9)$ & $29.0(7.5)$ & $-1.5(-2.1,-1.0)$ & $31.3(6.8)$ & $29.9(7.1)$ & $-1.4(-1.9,-1.0)$ & 0.77 \\
\hline Waist circumference, $\mathrm{cm}$ & $93.9(6.0)$ & $92.1(5.9)$ & $-1.8(-2.5,-1.1)$ & $94.1(5.1)$ & $92.7(5.1)$ & $-1.3(-2.1,-0.6)$ & 0.36 \\
\hline Hip circumference, $\mathrm{cm}$ & $100.2(4.7)$ & $99.3(4.2)$ & $-0.9(-1.4,-0.4)$ & $99.8(3.8)$ & $99.2(3.8)$ & $-0.6(-1.0,-0.3)$ & 0.42 \\
\hline Waist to hip ratio & $0.94(0.05)$ & $0.93(0.05)$ & $-0.01(-0.02,0.00)$ & $0.94(0.04)$ & $0.94(0.04)$ & $-0.01(-0.01,0.00)$ & 0.57 \\
\hline \multicolumn{8}{|c|}{ Participants whose step counts increased by fewer than 1,000 steps in 12 weeks } \\
\hline & \multicolumn{3}{|c|}{ A-mix group $(n=34)$} & \multicolumn{4}{|c|}{ Placebo group $(n=49)$} \\
\hline Abdominal total fat area, $\mathrm{cm}^{2}$ & $354.4(60.8)$ & $348.9(59.1)$ & $-5.4(-15.0,4.1)$ & $332.5(71.8)$ & $323.8(74.3)$ & $-8.7(-16.7,-0.7)$ & 0.60 \\
\hline $\begin{array}{l}\text { Abdominal subcutaneous fat } \\
\text { area, } \mathrm{cm}^{2}\end{array}$ & 270.I (54.4) & $269.7(52.9)$ & $-0.4(-9.2,8.4)$ & $236.9(71.1)$ & $233.9(71.4)$ & $-3.0(-9.8,3.9)$ & 0.64 \\
\hline Abdominal visceral fat area, $\mathrm{cm}^{2}$ & $84.2(30.9)$ & $79.2(33.6)$ & $-5.1(-9.4,-0.7)$ & $95.6(40.9)$ & $89.9(39.6)$ & $-5.7(-9.1,-2.4)$ & 0.80 \\
\hline Weight, kg & $71.8(8.7)$ & $71.5(8.9)$ & $-0.3(-0.9,0.2)$ & $72.7(7.6)$ & $71.7(7.6)$ & $-1.0(-1.6,-0.3)$ & 0.17 \\
\hline Body mass index, $\mathrm{kg} / \mathrm{m}^{2}$ & $27.3(1.3)$ & $27.1(1.3)$ & $-0.1(-0.4,0.1)$ & $27.2(1.4)$ & $26.9(1.5)$ & $-0.4(-0.6,-0.1)$ & 0.21 \\
\hline Percent fat mass, $\%$ & $34.4(6.6)$ & $33.8(6.6)$ & $-0.6(-1.0,-0.2)$ & $32.3(7.0)$ & $31.2(7.2)$ & $-1.0(-1.6,-0.5)$ & 0.24 \\
\hline Waist circumference, $\mathrm{cm}$ & $94.0(4.2)$ & $93.4(4.4)$ & $-0.7(-1.4,0.0)$ & $93.9(5.8)$ & $92.8(6.0)$ & $-1.1(-1.9,-0.3)$ & 0.24 \\
\hline Hip circumference, $\mathrm{cm}$ & $101.8(3.4)$ & $101.2(3.4)$ & $-0.6(-1.1,-0.1)$ & $100.4(3.5)$ & $99.9(3.6)$ & $-0.5(-1.0,0.0)$ & 0.78 \\
\hline Waist to hip ratio & $0.92(0.04)$ & $0.92(0.04)$ & $0.00(-0.01,0.00)$ & $0.94(0.05)$ & $0.93(0.05)$ & $-0.01(-0.01,0.00)$ & 0.25 \\
\hline
\end{tabular}

Notes: Data at weeks 0 and 12 are presented as mean (standard deviation). Changes from baseline to week 12 are presented as mean (95\% confidence interval). $P$-value is a result of unpaired $t$-test for 12-week change between groups.

study (Table S4). Therefore, it appears that the dose of 1,500 $\mathrm{mg}$ /day of A-mix supplementation had no cross-talk effect on BCAA metabolism.

The trial conducted in this study has some important strengths. First, this trial had a double-blind, placebocontrolled, and parallel-group randomized research design. Therefore, the findings are highly reliable. Second, the abdominal fat areas, as primary outcomes, were measured by $\mathrm{CT}$, which is considered to provide the highest quality assessment of abdominal fat. Third, the study included the promotion of physical activity by approximately 1,000 daily steps. This type of mild intervention, as opposed to an entirely regulated exercise program, can be easily adopted by the general population. ${ }^{18,19}$ Finally, the retention rate of the study was almost perfect (99\%).

There are also a few limitations of the study. First, we targeted overweight adults $\left(25 \mathrm{~kg} / \mathrm{m}^{2} \leq \mathrm{BMI}<30 \mathrm{~kg} / \mathrm{m}^{2}\right)$. Therefore, the effect of A-mix combined with physical activity promotion for obese adults $\left(\mathrm{BMI} \geq 30 \mathrm{~kg} / \mathrm{m}^{2}\right)$ was not investigated. Second, the changes in compliance outcomes, such as total and macronutrient intake, were different between the two groups; however, these between-group differences were not significant. Moreover, the energy intake of the test beverage is not included in this result. If it had been included, the difference of energy intake between the two groups would have been smaller.

\section{Conclusion}

The combination of A-mix supplementation and physical activity promotion facilitated abdominal fat reduction in overweight adults.

\section{Acknowledgments}

This trial was based on a collaborative research agreement between the University of Tsukuba Faculty of Medicine and Meiji Co., Ltd. Mr Takuya Nagai, Ms Fuyuko Yamamoto, and Mr Akio Tanaka produced and provided the test beverages used in this trial. Dr Naotaka Hashizume generated the randomization codes and created an allocation table. Ms Yuriko Sakairi provided administrative support and encouragement throughout the study. Data acquisition performed by staff from KSO Corporation. We gratefully acknowledge the contributions of all trial staff. Editorial support in the form of medical writing was provided by Editage. 


\section{Author contributions}

Study concept and design: KU, HS, CS, SI, and YN. Intervention: TT and YN. Statistical analysis: NS. Study physician: SS. Safety committee: HK. Interpretation: KU, HS, SS, and YN. Writing the first draft: KU. Overall supervision as a principal investigator: YN. All authors contributed toward revising the paper and agree to be accountable for all aspects of the work.

\section{Disclosure}

KU, CS, and SI are employees of Meiji Co., Ltd. The other authors HS, TT, HK, NS, SS, and YN report no conflicts of interest in this work.

\section{References}

1. Batsis JA, Nieto-Martinez RE, Lopez-Jimenez F. Metabolic syndrome: from global epidemiology to individualized medicine. Clin Pharmacol Ther. 2007;82(5):509-524.

2. Zou C, Shao J. Role of adipocytokines in obesity-associated insulin resistance. J Nutr Biochem. 2008;19(5):277-286.

3. Jensen MD, Ryan DH, Apovian CM, et al; American College of Cardiology/American Heart Association Task Force on Practice Guidelines; Obesity Society. 2013 AHA/ACC/TOS guideline for the management of overweight and obesity in adults: a report of the American College of Cardiology/American Heart Association Task Force on Practice Guidelines and The Obesity Society. Circulation. 2014;129(25 Suppl 2):S102-S138.

4. Kim HK, Suzuki T, Saito K, et al. Effects of exercise and amino acid supplementation on body composition and physical function in community-dwelling elderly Japanese sarcopenic women: a randomized controlled trial. J Am Geriatr Soc. 2012;60(1):16-23.

5. Michishita T, Kobayashi S, Katsuya T, Ogihara T, Kawabuchi K. Evaluation of the antiobesity effects of an amino acid mixture and conjugated linoleic acid on exercising healthy overweight humans: a randomized, double-blind, placebo-controlled trial. J Int Med Res. 2010;38(3):844-859.

6. Abe T, Takiguchi Y, Tamura M, Shimura J, Yamazaki K. Effect of amino acid mixture (VAAM) isolated from larval saliva and modified VAAM nutrients on endurance exercise in swimming mice-improvement in performance and changes of blood lactate and glucose. Jpn J Phys Fitness Sports Med. 1995;44(2):225-238.

7. Abe T, Inamori M, Iida K, Tamura M, Takiguchi Y, Yasuda K. The activation of fatty acid metabolism by vespa amino acid mixture (VAAM) and related nutrients during endurance exercise in mice. Adv Exerc Sports Physiol. 1997;3(1):35-44.

8. Tsuchita H, Shirai-Morishita Y, Shimizu T, Abe T. Effects of a vespa amino acid mixture identical to hornet larval saliva on the blood biochemical indices of running rats. Nutr Res. 1997;17(6):999-1012.

9. Mizuno K, Asano K, Abe T, Morishita K. Effects of ingestion of hornet larval salivary amino acid mixture (HLSA) on metabolic responses during exercise. Sci Fatigue Rest. 1997;12(1):31-41. Japanese.

10. Saito $S$, Tsuchita H, Mukai N, Abe T. Effects of ingestion of vespa amino acid mixture (VAAM) under postprandial conditions on blood ketone body concentrations during prolonged exercise in humans. Bull Inst Health Sport Sci Univ Tsukuba. 2001;24:71-78. Japanese.

11. Sasai H, Matsuo T, Fujita M, Saito M, Tanaka K. Effects of regular exercise combined with ingestion of vespa amino acid mixture on aerobic fitness and cardiovascular disease risk factors in sedentary older women: a preliminary study. Geriatr Gerontol Int. 2011;11(1):24-31.

12. Ueda K, Nakamura Y, Yamaguchi M, Mori T, Uchida M, Fujita S. Amino acid mixture enriched with arginine, alanine, and phenylalanine stimulates fat metabolism during exercise. Int J Sport Nutr Exerc Metab. 2016;26(1):46-54.
13. Ueda K, Sanbongi C, Takai S, Ikegami S, Fujita S. Combination of aerobic exercise and an arginine, alanine, and phenylalanine mixture increases fat mobilization and ketone body synthesis. Biosci Biotechnol Biochem. 2017;81(7):1417-1424.

14. Ueda K, Sanbongi C, Ikegami S. An arginine, alanine, and phenylalanine mixture increases synthesis of ketone bodies during low-intensity exercise via stimulating glucagon secretion in men with obesity. $J$ Phys Fit Sports Med. 2017;6(5):325-333.

15. Ueda K, Sanbongi C, Yamaguchi M, Ikegami S, Hamaoka T, Fujita $\mathrm{S}$. The effects of phenylalanine on exercise-induced fat oxidation: a preliminary, double-blind, placebo-controlled, crossover trial. J Int Soc Sports Nutr. 2017;14:34.

16. Sasai H, Ueda K, Tsujimoto T, et al. Dose-ranging pilot randomized trial of amino acid mixture combined with physical activity promotion for reducing abdominal fat in overweight adults. Diabetes Metab Syndr Obes. 2017;10:297-309.

17. Schulz KF, Altman DG, Moher D; CONSORT Group. CONSORT 2010 statement: updated guidelines for reporting parallel group randomized trials. Ann Intern Med. 2010;152(11):726-732.

18. Miyachi M, Tripette J, Kawakami R, Murakami H. “+10 min of physical activity per day": Japan is looking for efficient but feasible recommendations for its population. J Nutr Sci Vitaminol (Tokyo). 2015;61(Suppl):S7-S9.

19. Murakami H, Tripette J, Kawakami R, Miyachi M. "Add 10 min for your health": the new Japanese recommendation for physical activity based on dose-response analysis. J Am Coll Cardiol. 2015;65(11): $1153-1154$

20. Kurotani K, Akter S, Kashino I, et al; Japan Public Health Center based Prospective Study Group. Quality of diet and mortality among Japanese men and women: Japan Public Health Center based prospective study. BMJ. 2016;352:i1209.

21. Shafer KJ, Siders WA, Johnson LK, Lukaski HC. Validity of segmental multiple-frequency bioelectrical impedance analysis to estimate body composition of adults across a range of body mass indexes. Nutrition 2009;25(1):25-32.

22. Yoshizumi T, Nakamura T, Yamane M, et al. Abdominal fat: standardized technique for measurement at CT. Radiology 1999;211(1):283-286.

23. Oshima Y, Kawaguchi K, Tanaka S, et al. Classifying household and locomotive activities using a triaxial accelerometer. Gait Posture. 2010;31(3):370-374.

24. Ohkawara K, Oshima Y, Hikihara Y, Ishikawa-Takata K, Tabata I, Tanaka S. Real-time estimation of daily physical activity intensity by a triaxial accelerometer and a gravity-removal classification algorithm. Br J Nutr. 2011;105(11):1681-1691.

25. Troiano RP, Berrigan D, Dodd KW, Mâsse LC, Tilert T, McDowell M. Physical activity in the United States measured by accelerometer. Med Sci Sports Exerc. 2008;40(1):181-188.

26. Gannon MC, Nuttall FQ. Amino acid ingestion and glucose metabolism-a review. IUBMB Life. 2010;62(9):660-668.

27. Blecher M, Merlino NS, Ro'Ane JT, Flynn PD. Independence of the effects of epinephrine, glucagon, and adrenocorticotropin on glucose utilization from those on lipolysis in isolated rat adipose cells. $J$ Biol Chem. 1969;244(13):3423-3429.

28. Holst JJ, Christensen M, Lund A, et al. Regulation of glucagon secretion by incretins. Diabetes Obes Metab. 2011;13(Suppl 1):89-94.

29. Elam RP. Morphological changes in adult males from resistance exercise and amino acid supplementation. J Sports Med Phys Fitness. 1988;28(1):35-39.

30. Schena F, Guerrini F, Tregnaghi P, Kayser B. Branched-chain amino acid supplementation during trekking at high altitude. The effects on loss of body mass, body composition, and muscle power. Eur J Appl Physiol Occup Physiol. 1992;65(5):394-398.

31. Mourier A, Gautier JF, De Kerviler E, et al. Mobilization of visceral adipose tissue related to the improvement in insulin sensitivity in response to physical training in NIDDM. Effects of branched-chain amino acid supplements. Diabetes Care. 1997;20(3):385-391. 
32. Antonio J, Sanders MS, Ehler LA, Uelmen J, Raether JB, Stout JR. Effects of exercise training and amino-acid supplementation on body composition and physical performance in untrained women. Nutrition. 2000;16(11-12):1043-1046.

33. Lucotti P, Setola E, Monti LD, et al. Beneficial effects of a long-term oral L-arginine treatment added to a hypocaloric diet and exercise training program in obese, insulin-resistant type 2 diabetic patients. Am J Physiol Endocrinol Metab. 2006;291(5):E906-E912.

34. Kendrick IP, Harris RC, Kim HJ, et al. The effects of 10 weeks of resistance training combined with beta-alanine supplementation on whole body strength, force production, muscular endurance and body composition. Amino Acids. 2008;34(4):547-554.

35. Ispoglou T, King RF, Polman RC, Zanker C. Daily L-leucine supplementation in novice trainees during a 12-week weight training program. Int J Sports Physiol Perform. 2011;6(1):38-50.

36. Forbes SC, Harber V, Bell GJ. The acute effects of L-arginine on hormonal and metabolic responses during submaximal exercise in trained cyclists. Int J Sport Nutr Exerc Metab. 2013;23(4):369-377.
37. Romijn JA, Coyle EF, Sidossis LS, et al. Regulation of endogenous fat and carbohydrate metabolism in relation to exercise intensity and duration. Am J Physiol. 1993;265 (3 Pt 1):E380-E391.

38. Kanemitsu H, Yamamoto T, Kemi M, et al. Safety evaluation of A-mix (4)single-dose oral toxicity study in rats. Jpn Pharmacol Ther. 2017;45(4): 531-533. Japanese.

39. Kanemitsu H, Yamamoto T, Nakamura D, et al. Safety evaluation of A-mix (5) - 13-week oral toxicity study in rats. Jpn Pharmacol Ther. 2017;45(4):535-548. Japanese.

40. Minekawa K, Fujiwara N, Nakamura K, et al. Safety evaluation of A-mix (1) - Bacterial reverse mutation test. Jpn Pharmacol Ther. 2017;45(4): 515-519. Japanese.

41. Fischer JE, Yoshimura N, Aguirre A, et al. Plasma amino acids in patients with hepatic encephalopathy. Effects of amino acid infusions. Am J Surg. 1974;127(1):40-47.
Diabetes, Metabolic Syndrome and Obesity: Targets and Therapy is an international, peer-reviewed open-access journal committed to the rapid publication of the latest laboratory and clinical findings in the fields of diabetes, metabolic syndrome and obesity research. Original research, review, case reports, hypothesis formation, expert opinion and commentaries are all considered for publication. The manuscript management system is completely online and includes a very quick and fair peer-review system, which is all easy to use. Visit http://www.dovepress.com/testimonials.php to read real quotes from published authors.

Submit your manuscript here: https://www.dovepress.com/diabetes-metabolic-syndrome-and-obesity-targets-and-therapy-journal 